\title{
The influence of vaginal ovules on vaginal microbiome and vulvovaginitis
}

\author{
Nicolae Bacalbasa ${ }^{1,2}$, Irina Balescu ${ }^{3}$, Adnan Ad Aloul ${ }^{4,5}$, Roxana Bohiltea ${ }^{1,6}$, Bogdan Socea ${ }^{7,8}$, \\ Bogdan Ursut ${ }^{1,9}$, Alexandru Filipescu ${ }^{1,10}$ \\ ${ }^{1}$ Department of Obstetrics and Gynecology, "Carol Davila" University of Medicine and Pharmacy, Bucharest, Romania \\ ${ }^{2}$ Department of Visceral Surgery, Center of Excellence in Translational Medicine, Fundeni Clinical Institute, \\ Bucharest, Romania \\ ${ }^{3}$ Department of Visceral surgery, Ponderas Academic Hospital, Bucharest, Romania \\ ${ }^{4}$ Department of Surgery, Ramnicu Sarat County Hospital, Buzau, Romania \\ ${ }^{5}$ Department of Surgery, "Titu Maiorescu" University, Bucharest, Romania \\ ${ }^{6}$ Department of Obstetrics and Gynecology, University Emergency Hospital, Bucharest, Romania \\ ${ }^{7}$ Department of Surgery, "Carol Davila" University of Medicine and Pharmacy, Bucharest, Romania \\ ${ }^{8}$ Department of Surgery, "Sf. Pantelimon" Clinical Emergency Hospital, Bucharest, Romania \\ ${ }^{9}$ Department of Surgery, "Agrippa Ionescu" Clinical Emergency Hospital, Bucharest, Romania \\ ${ }^{10}$ Department of Obstetrics and Gynecology, Elias Emergency Hospital, Bucharest, Romania
}

Vaginal microbiome is submitted to permanent changes accordingly to age, menopausal status or association of different pathological conditions such as inflammation or mucosal atrophy. The presence of these modifications is usually associated with local development of infectious, inflammatory or atrophic vulvovaginitis. These represent the most commonly complaints which affect women at all ages. Therefore, attention was focused on creating a topic product which is able to control the local process and to alleviate the symptoms. The aim of the current paper is to analyse the physiology, physiopathology and therapeutic strategies in such cases with special focus on Cerviron, a product which seems to provide multiple therapeutic benefits in such cases.

Keywords: vaginal microbioma, vulvovaginitis, Cerviron

\section{INTRODUCTION}

Vaginal microbiome represents a dynamic microecosystem which is permanently submitted to changes during the different phases of the menstrual cycle as well as during women's entire life; therefore, it is widely demonstrated that before menarche and after menopause onset similar features of the vaginal microbiome are encountered, which are significantly different when compared to those reported during the reproductive age (1). One of the most important components of the vaginal microbiome is represented by lactobacilli, which are able to utilize glycogen - directly controlled by oestrogens - and to produce lactic acid, one of the most important chemical substances which confer the ability to prevent the development of certain infections. Therefore, it is widely demonstrated that before menarche and after the menopausal onset the levels of oestrogen decrease and so does the level of glycogen deposits; in this respect the amount of lactic acid decreases and therefore the vaginal $\mathrm{pH}$ increases, leading to the apparition of a basic habitat which will further favour the apparition of pathogenic bacteria (1-5). Meanwhile, non-lactobacillary 
flora is present, the type and rate of the microbiota being significantly influenced by race. Whenever the number of lactobacilli decrease, the risk of apparition of other microorganisms significantly increase, leading to the apparition of bacterial vaginosis (2,7-10). Furthermore, this condition seems to be directly correlated with the apparition of other significant disorders such as pelvic inflammatory disease, human immunodeficiency virus infection, human papilloma virus infection or adverse outcomes during pregnancy (3).

\section{NOTIONS OF PHYSIOLOGY}

The vaginal mucosa presents a stratified squamous nonkeratinized epithelium and is covered by a cervico-vaginal secretion; however, due to the limited blood supply, the vaginal mucosa acquires oxygen and nutrients through diffusion establishing in this way a partially anaerobic medium (4). Lactobacillus species are found in this anaerobic habitat and seem to have the capacity to produce different antimicrobial products such as lactic acid, bacteriocins and hydrogen peroxide creating in this way an acid environment, with a pH lower than 4,5 and preventing in this way the further development of various infections $(4,5)$.

\section{BACTERIAL VAGINOSIS}

Once the rates of lactobacillus decrease the local concentration of lactic acid and peroxide will decrease and therefore the vaginal $\mathrm{pH}$ will increase, creating in this way the proper conditions for the development of facultative or obligate anaerobic bacteria such as Prevotella or Gardnerella and therefore for the apparition of bacterial vaginosis. Furthermore, this condition seems to be strongly associated with severe conditios such as preterm birth, pelvic inflammatory disease, human papilloma infection, low birtweight or even miscarriage; in this respect it can be widely explained the fact that this should be considered as a serious condition and should be treated in consequence. Therefore, it should not be omitted the fact that human papilloma virus infection represents the leading cause of severe pathological conditions such as cervical intraepithelial neoplasia ann cervical cancer as well as vaginal and vulvar cancer. In this respect, attention should be focused on preventing the development of vaginosis (6-8).

\section{FACTORS INFLUENCING THE COMPOSITION OF NORMAL VAGINAL MICROBIOMA}

As mentioned before, the composition of the vaginal microbioma is influenced by the patient's age and hormonal status; therefore, while in young girls the predominant flora is represented by lactobacillus in association with Staphylococcus, Streptococcus and Gram positive bacilli, in adults this flora is dominated by lactobacillus, anaerobic bacilli, enterobacter, Staphylococcus and Streptococcus while in postmenopausal women vaginal flora is more likely to the one reported before menarche $(9,10)$.

The most important environmental factors which seem to influence the composition of vaginal microbioma are represented by the excessive use of antibiotics, diet, pregnancy, vaginal douches, using intrauterine devices of contraception and wet and warm climate; meanwhile it seems that increased serum levels of oestrogens (such as those which have been encountered during pregnancy, in cases receiving hormonal substitution therapy or in cases receiving long term prednisone based treatment).

\section{TYPES OF VAGINOSES}

As stated before, once the number of lactobacillus decreases, the vagional $\mathrm{pH}$ will increase and will create the proper environment for the development of other microorganisms such as Gardnerella vaginalis or different types of Streptococcus leading to the apparition of endogen vaginal infections. Meanwhile transmitted sexual infections are also responsible for the apparition of other types of vaginosis, also called exogen vaginal infections; the most commonly incriminated germs are represented by herpes simplex virus type 2, human papilloma virus, Chlamydia trachomatis, Neisseria gonorrhoea, Trichomonas vaginalis and fungi.

When it comes to the clinical manifestations of this pathological condition, two different types of vaginosis should be separately described. Therefore, the most commonly encountered type is the inflammatory one, characterised by the presence of local inflammation such as local oedema and reddish aspect of the vulvo-vaginal area. The other type is represented by atrophic vaginitis and is rather related to deficiency of oestrogen, being more frequently encountered in postmenopausal women. In such cases the vaginal mucosa is thinner, reduced in elasticity and dry. In these conditions, local symptoms such as itching, burn sensation and pain during sexual intercourse are frequently reported. However, in certain cases reddish aspect as well as purulent discharge can be encountered, the final differential diagnostic being established at colposcopy; therefore, the thinner aspect of the vaginal lining should be considered as a definitive criteria.

\section{THERAPEUTIC STRATEGIES IN VULVOVAGINITIS}

Due to the fact that vulvovaginitis is most often caused by local inflammation and infection, the 
most important therapeutic strategies are represented by local administration of topic products in order to re-establish the normal microbiota, to recreate an acid environment and to diminish the local inflammation. Meanwhile, due to the fact that multiple causes might be incriminated, treatment should be targeted at the specific aetiology which is incriminated in each case (11). Whenever an infectious cause is suspected, microbiological analysis is needed in order to identify the pathogenic agent and further to continue the treatment accordingly. Depending on the extent of the disease and the general status of the patient, local or systemic treatment might be recommended (12-14).

A recent product which seems to provide promising results in such cases is represented by Cerviron. This is an intravaginal agent which includes seven active products; these components seem to provide multiple benefits due to the fact that they are able to re-establish a normal vaginal microbioma, a normal vaginal $\mathrm{pH}$ as well as to provide an efficient local anti-inflammatory, antibacterian and antiifungical effect; meanwhile it provides an efficient healing effect of all local microlesions. Cerviron represents an association of three topical pharmaceutical products - hexylresorcinol, collagen and bismuth subgallate and four phyto products: calendula extract, Hydrastis extract, Thymus vulgaris extract and Curcuma extract.

Hexylresorcinol represents an efficient antiseptic, antimicrobian and antihelmintic product which seems to provide an efficient local effect against pathogenic products of different vaginal bacteria; meanwhile it seems to increase the efficacy of systemic antibiotic therapy, to decrease the length of the systemic antibiotic treatment and therefore to diminish the risk of developing bacterial resistance (15).

Collagen, another active product which is part of Cerviron, is widely recognised for its' healing capacity of different wounds, especially in the case of non-infected lesions; therefore, topic administration of collagen is able to provide an effective healing of the vaginal microlesions of the mucosa and to create a protective pellicle at this level (16).

Bismuth subgallate is a hemostatic agent which creates an efficient, insoluble pellicle surface which provides a more rapid healing of the local wounds, it diminishes the risk of suprainfection of the wound and diminishes the risk of formation of an excessive granular tissue (17).

As for the phyto components of Cerviron, Calendula officinalis, Thymus vulgaris and Curcuma are widely recognised for their antiseptic and healing properties increasing therefore the efficacy of the other pharmacologically active components (18).

In order to demonstrate and investigate the efficacy of this product, there are two on going prospective studies (CYRON/01/2021 and CYRON/02/2021) which will include 50 patients each and which will be ended at the beginning of the year of 2022. The first study has as primary end points the reestablishment of the normal vaginal microbioma as well as of the normal vaginal $\mathrm{pH}$ in the setting of daily administration of this product for three months. The second study will aim to investigate the efficacy of the product in patients submitted to local excision of cervical lesions in the absence of human papilloma virus infection.

Data obtained in these studies will further orientate the therapeutic strategies in such cases and will demonstrate which are the symptoms and signs which are significantly improved by using this product.

\section{CONCLUSIONS}

Vaginal microbioma is permanently submitted to changes, it's composition being widely influenced by different factors. In these conditions once the biological equilibrium is destroyed, local modifications such as inflammation, bacterial, fungal or even parasite infections can occur and can lead to the apparition of different types of vulvo-vaginitis. Meanwhile in postmenopausal women decreased amounts of oestrogen will lead to the apparition of vaginal mucosa atrophy, predisposing in this way to the apparition of atrophic vaginitis. In this respect attention was focused in creating a product which contains multiple active principles and which is able to confer an antibacterial, antifungical and anti-inflammatory effect; meanwhile collagen and plant derived products increase the healing effect and improve the local vascularization improving in this way the features of the vaginal lining.

\section{Conflict of interest: none declared} Financial support: none declared

\section{REFERENCES}

1. Buchta V. Vaginal microbiome. Ceska Gynekol. 2018;83(5):371-379.

2. Chen X, Lu Y, Chen T, Li R. The Female Vaginal Microbiome in Health and Bacterial Vaginosis. Front Cell Infect Microbiol. 2021 April.

3. Fredricks DN, Fiedler TL, Marrazzo JM. Molecular identification of bacteria associated with bacterial vaginosis. N Engl J Med. 2015;353:1899-1911.
4. Linhares, IM, Summers PR, Larsen B, Giraldo PC, Witkin SS. Contemporary perspectives on vaginal $\mathrm{pH}$ and lactobacilli. Am J Obstet

5. Witkin SS, Mendes-Soares H, Linhares IM, Jayaram A, Ledger WJ, Forney $L$ J. Influence of vaginal bacteria and D- and L-lactic acid isomers on vaginal extracellular matrix metalloproteinase inducer: implications Gynecol. 2011;204(2):120. 
for protection against upper genital tract infections. mBio. 2013;4(4):00460-00413.

6. Hillier SL, Nugent RP, Eschenbach DA, Krohn MA, et al. Association between bacterial vaginosis and preterm delivery of a low-birth-weight infant. The Vaginal Infections and Prematurity Study Group. N Engl J Med. 1995;333:1737-1742.

7. Klatt NR, Cheu R, Birse K, Zevin AS, Perner M, et al. Vaginal bacteria modify HIV tenofovir microbicide efficacy in African women. Science. 2017;356:938-945.

8. Leitich $\mathrm{H}$, Kiss $\mathrm{H}$. Asymptomatic bacterial vaginosis and intermediate flora as risk factors for adverse pregnancy outcome. Best Pract Res Clin Obstet Gynaecol. 2007;21:375-390.

9. Devillard E, Burton JP, Hammond J-A, Lam D, Reid G. Novel insight into the vaginal microflora in postmenopausal women under hormone replacement therapy as analyzed by PCR-denaturing gradient gel electrophoresis. Eur J Obstet Gynecol Reprod Biol. 2004 Nov 10;117(1):76-81.

10. Hillier SL, Lau RJ. Vaginal microflora in postmenopausal women who have not received estrogen replacement therapy. Clin Infect Dis Off Publ Infect Dis Soc Am. 1997 Sep;25 Suppl 2:S123-126.

11. Zuckerman A, Romano M. Clinical Recommendation: Vulvovaginitis. J Pediatr Adolesc Gynecol. 2016;29: 673-679.

12. McClelland R, Richardson B, Hassan W, Graham Sm, Kiarie J, Baeten JM, Mandaliya K, Jaoko W, Ndinya-Achola JO, Holmes KK. Prospective study of vaginal bacterial flora and other risk factors for vulvovaginal candidiasis. J Infect Dis. 2009;199(12):1883-1890.

13. Workowski K, Bolan G. Centers for Disease Control and Prevention. Sexually transmitted diseases treatment guidelines, 2015. Practice Guideline. MMWR Recomm Rep. 2015;5:1-137.

14. Bradshaw C, Morton A, Hocking J, Garland SM, Morris MB, et al. High recurrence rates of bacterial vaginosis over the course of 12 months after oral metronidazole therapy and factors associated with recurrence. J Infect Dis. 2006; 193:1478-1486.

15. Nikolaev YA, Tutel'yan AV, Loiko NG, Buck J, et al. The use of 4-Hexylresorcinol as antibiotic adjuvant. PloS One. 2020 Sep 22;15(9):e0239147.

16. Verstraelen $H$, Swidsinski A. The biofilm in bacterial vaginosis: implications for epidemiology, diagnosis and treatment. Curr Opin Infect Dis. 2013; 26:86-89.

17. Sobel JD, Chaim W, Nagappan V, Leaman D. Treatment of vaginitis caused by Candida glabrata: use of topical boric acid and flucytosine. Am J Obstet Gynecol. 2003;189:1297-300.

18. Bosserman E, Helms D, Mosure D, Secor WE, Workowski KA. Utility of antimicrobial susceptibility testing in Trichomonas vaginalis-infected women with clinical treatment failure. Sex Transm Dis. 2011;38(10): 983-987. 